\title{
BMJ Open Impact of a compression garment, on top of the usual care, in patients with breast cancer with early disturbance of the lymphatic transport: protocol of a randomised controlled trial
}

\author{
Sarah Thomis (D) , ${ }^{1,2}$ Nele Devoogdt, ${ }^{1,3}$ Beate Bechter-Hugl, ${ }^{1}$ Ines Nevelsteen, ${ }^{4}$ \\ Patrick Neven, ${ }^{4}$ Inge Fourneau ${ }^{1,2}$
}

To cite: Thomis S, Devoogdt N, Bechter-Hugl B, et al. Impact of a compression garment, on top of the usual care, in patients with breast cancer with early disturbance of the lymphatic transport: protocol of a randomised controlled trial. BMJ Open 2020;10:e042018. doi:10.1136/ bmjopen-2020-042018

- Prepublication history for this paper is available online To view these files, please visit the journal online (http://dx.doi org/10.1136/bmjopen-2020042018).

Received 24 June 2020 Revised 27 October 2020 Accepted 09 November 2020

Check for updates

(C) Author(s) (or their employer(s)) 2020. Re-use permitted under CC BY-NC. No commercial re-use. See rights and permissions. Published by BMJ.

For numbered affiliations see end of article.

Correspondence to

Dr Sarah Thomis;

sarah.thomis@uzleuven.be

\section{ABSTRACT}

Introduction Breast cancer-related lymphoedema (BCRL) is a common phenomenon. When lymphoedema is diagnosed late, options for treatment are diminished. Therefore, early diagnosis and treatment are very important to alter the potential deleterious evolution. Lymphofluoroscopy visualises the superficial lymphatic architecture in detail, giving the opportunity to detect a disturbance in the lymphatic transport (ie, dermal backflow) before the lymphoedema is clinically visible. The main objective is to investigate if there is an additional effect of a compression garment on top of the usual care (ie, information and exercises) in patients with early disturbance of the lymphatic transport after breast cancer treatment. Development of clinical lymphoedema and/ or deterioration of the dermal backflow visualised by lymphofluoroscopy is investigated.

Methodology All patients scheduled for breast cancer surgery with unilateral axillary lymph node dissection or sentinel node biopsy in the Multidisciplinary Breast Clinic of the University Hospitals Leuven are being considered. Patients are assessed before surgery and at 1, 3, 6, 9, $12,18,24$ and 36 months postoperatively. At each visit, a clinical assessment is performed determining the volume difference between both arms and hands (through circumference measurements and water displacement), the water content, the extracellular fluid, the pitting status and the skinfold thickness. Quality of life questionnaires are filled in. At each visit, a lymphofluoroscopy is performed as well. When a disturbance of the lymphatic transport is seen on lymphofluoroscopy, without the presence of clinical lymphoedema, the patient is randomised in either a control group receiving usual care or a preventive treatment group receiving usual care and a compression garment (whether or not combined with a glove).

Ethics and dissemination The trial is conducted in compliance with the principles of the Declaration of Helsinki (2008), the principles of Good Clinical Practice and in accordance with all applicable regulatory requirements. This protocol has been approved by the Ethical Committee of the University Hospitals Leuven. Results will be disseminated by peer-reviewed scientific journals and presentation at international congresses.
Strengths and limitations of this study

- This is the first study to investigate the additional effect of early treatment in patients with breast cancer with a disturbance on lymphofluoroscopy.

- This is a prospective, randomised controlled trial.

- Lymphofluoroscopy and clinical measurements are performed preoperative and at regular times up to 3 years after surgery for breast cancer.

- This study is powered for the primary outcomes incidence of clinical lymphoedema and deterioration of dermal backflow.

Trial registration number NCT03210311

Conclusion The investigators hypothesise that development of clinical BCRL can be prevented and/or the dermal backflow can be stabilised or improved, if a preventive treatment with compression garment is started in the early phase of disturbance.

\section{INTRODUCTION}

lymphoedema is a chronic and debilitating disease caused by imbalance between lymph production and lymph transport. It reduces patient's quality of life by limb enlargement but also by other physical and psychosocial problems, for example, decreased mobility, recurrent infections, stress and decreased ability to perform occupational activities. ${ }^{1-3}$

Breast cancer-related lymphoedema (BCRL) is a secondary lymphoedema of the upper limb that can occur after treatment for breast cancer. Incidence of BCRL vary in literature, especially since the introduction of less invasive techniques such as sentinel node procedures and radiotherapy. According to a review of DiSipio et al, the incidence of arm lymphoedema was about four times higher in women who had an axillary lymph node dissection (ALND) (19.9\%) than after 
sentinel lymph node biopsy (5.6\%). ${ }^{45}$ A study by Rockson suggested that in almost $75 \%$ of the cases, lymphoedema is established within the first year after breast cancer treatment. ${ }^{6}$ A volume difference between both limbs of $5 \%-10 \%$ is normally used to define clinical lymphoedema $^{47}$

Lymphoedema can progress from a soft pitting oedema to a hard fibrotic or soft fatty and non-pitting oedema because of lipogenesis, fibrosis, inflammation, lymphangiogenesis and immunosuppression. ${ }^{89}$

There is no consensus concerning the best measuring tool to detect the development of BCRL. ${ }^{10}{ }^{11}$ Volume increase of the limb can be assessed with circumference measurements ${ }^{12}$ or with the water displacement method. ${ }^{13}{ }^{14}$ A relative volume change between both arms is used, comparing preoperative measurements between the affected arm and the healthy arm, to the postoperative measurements. ${ }^{7}$ In addition, the increase of water content in the edematous limb can be assessed by the pitting test, ${ }^{15}$ by measuring the extracellular fluid (bioelectrical impedance spectroscopy) ${ }^{16}$ or by measuring the water content of the skin (tissue dielectric constant). ${ }^{1718}$ Measurement of the skinfold thickness (Stemmer sign) can be performed, which is the typical sign for lymphoedema. ${ }^{19}$

Historically lymphangiography has been the technique to image the lymphatic system. This technique is difficult to perform and has become obsolete. ${ }^{20}$ Lymphoscintigraphy has replaced lymphangiography and became the new standard for imaging the lymphatic system. With lymphoscintigraphy a radionuclide $\quad\left({ }^{99 \mathrm{~m}} \mathrm{Tc}\right.$-labelled tracer) is injected and followed by sequential gamma imaging. ${ }^{212}$ This technique not only provides dynamic imaging of the lymphatics and the lymph nodes, but also provides semiquantitative data of radionuclide transport and lymph node absorption. Near-infrared fluorescence imaging or lymphofluoroscopy is another minimally invasive technique. The injection of indocyanine green (ICG) intradermally allows to visualise lymphatics in the upper $2 \mathrm{~cm}$ of the skin using an infrared camera system, capturing the fluorescence. ${ }^{2324}$ It provides real-time relatively high-resolution images and detailed information about the superficial lymphatic transport. ${ }^{25}$ The images themselves are classified in different patterns: a normal linear lymph transport pattern and three dysfunctional dermal backflow (DB) patterns. The first dysfunctional pattern is the splash pattern, representing a dispersed tracer in tortuous lymphatic channels. The second is the stardust pattern, which demonstrates spotted fluorescent signals, representing the effusion of lymph fluid into the interstitium. The last type is the diffuse pattern wherein the tracer is widely distributed without identifiable spots. In this pattern, besides accumulation in the lymphatic capillaries and lymph precollectors, lymph stagnates in the interstitium. ${ }^{25}{ }^{26}$ Different studies have demonstrated that lymphofluoroscopy is a valid imaging technique to evaluate superficial lymphatic transport in patients with BCRL ${ }^{27} 28$ and can be used for early detection of BCRL. ${ }^{29}$
To prevent further evolution to fibrous and fatty tissue, early start of BCRL treatment is recommended. ${ }^{30}{ }^{31}$ Previous studies demonstrated that early detection of BCRL with clinical measurement tools such as bioelectrical impedance spectroscopy and volume measurements and subsequently early start of manual lymph drainage and exercise, reduces the rate of clinical lymphoedema. ${ }^{32}{ }^{33}$ Encouraging participation in regular exercise and maintaining healthy body weight as well as giving information such as avoiding infection, heat and tight clothing are guidelines to prevent lymphoedema. ${ }^{34}$ The previous studies investigated the effect of early treatment by using clinical assessments. The optimal tool to use remains unclear, and furthermore patient subjective symptoms and extremity volume can vary depending on the timing of measurement (morning and evening), the temperature, the activities performed by the patient during the day, ${ }^{35}{ }^{36}$ thus not reliable for lymphoedema diagnosis. Subclinical lymphoedema should be diagnosed with lymphatic imaging.

Therefore, the aim of this study is to investigate the additional effect of wearing a compression garment on top of the usual care (ie, exercise and information), on the incidence of clinical lymphoedema and/or deterioration of the DB visualised by lymphofluoroscopy, in patients developing early disturbance after treatment for breast cancer.

\section{METHODOLOGY \\ Trial design}

This study is a prospective randomised controlled trial. Figure 1 gives an overview of the participant flow in the trial. All participants are assessed at the Department of Vascular Surgery of the University Hospitals Leuven. The trial started in November 2017 and will end in May 2023.

\section{Patient and public involvement}

The protocol was discussed extensively with the oncologists of the Multidisciplinary Breast Clinic. Patients with BCRL were involved in the trial design and the methods of assessing the lymphoedema. They were informed through information sessions at the centre for lymphoedema. The results of the study will be communicated in a symposium organised for patients recruited in the study and the patients whom were involved in the trial design.

\section{Participants}

All patients scheduled for breast surgery combined with either unilateral ALND or sentinel node biopsy (SNB) in the multidisciplinary breast clinic at the University Hospitals Leuven are screened for participation in the study.

Recruitment started in November 2017. Inclusion criteria were: (1) age $\geq 18 y$, (2) women/men with breast cancer and scheduled for unilateral ALND or SNB, (3) oral and written approval of informed consent, (4) understanding Dutch. Exclusion criteria were (1) oedema of the upper limb from other causes, (2) cannot participate 


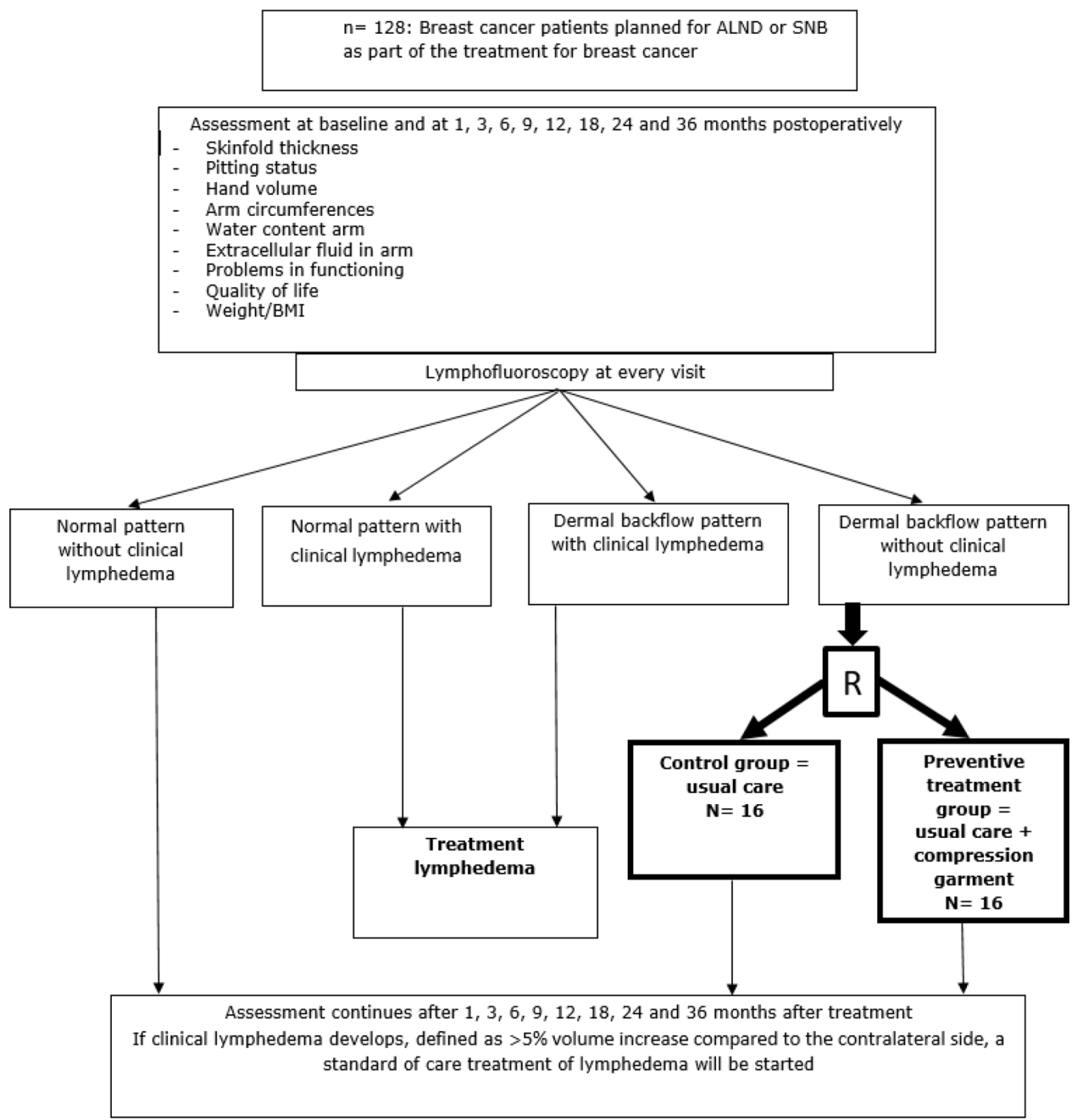

Figure 1 Flow of participants in the trial. ALND, axillary lymph node dissection; SNB, sentinel node biopsy.

during the entire study period, (3) mentally or physically unable to participate in the study, (4) contraindication for the use of ICG: allergy to ICG, iodine, hyperthyroidism and (5) metastatic disease.

All patients receive written as well as oral information. All included patients sign an informed consent document prior to the start of the study.

\section{Assessments}

Figure 1 gives an overview of the different assessments and their timing in the trial. All assessments are performed at baseline and at 1, 3, 6, 9, 12, 18, 24 and 36 months postoperatively.

\section{NEAR-INFRARED FLUORESCENCE IMAGING OF THE LYMPHATIC SYSTEM OR}

\section{LYMPHOFLUOROSCOPY}

All lymphofluoroscopic assessments are performed by one person (ST) who is blinded to the participant's data as well as to the assigned group if relevant.

During lymphofluoroscopy, ICG is injected intradermally in the first and fourth webspace of the hand on the affected side. An infrared camera system (Photo Dynamic Eye (PDE) camera, Hamamatsu®) captures the fluorescence. The procedure consists of three consecutive phases (table 1): an early phase, a break and a late phase.
All information about the lymphatic transport is documented in a standard evaluation document and in case of disturbance, this information is drawn on a body diagram according to the legend (figure 2).

\section{CLINICAL ASSESSMENTS}

The clinical assessments are performed by one assessor. In order to ensure blinding of the assessor, participants are asked not to share any information concerning their treatment (eg, wearing compression garment or not) neither to wear their compression material during evaluations. In addition, the assessor is blinded to previous measurement data in order to avoid being influenced by previous results.

Tables 2 and 3 provide a detailed overview of the clinical evaluation methods and procedures performed. Figure 3 shows the reference points used for the local clinical assessments.

\section{Randomisation and allocation sequence generation}

After visualisation of an early disturbance of the lymphatic transport, without the presence of clinical lymphoedema, patients are randomised in either the control group or the preventive treatment group. Randomisation is performed 
Table 1 Protocol near-infrared fluorescence imaging

\begin{tabular}{lll}
\hline Step & Description & Reporting \\
\hline Preparation & 0.1 Dilution of ICG & $\begin{array}{l}\text { Suspended ICG in } 25 \mathrm{~mL} \text { pure water and } \\
\text { subsequently diluted with saline water to } \\
\text { reach a final concentration of } 0.20 \mathrm{mg} / \mathrm{mL}\end{array}$ \\
& 0.2 Camera & $\begin{array}{l}\text { Camera is held perpendicular to the } \\
\text { observed skin at distance of } 15 \mathrm{~cm} \text { (best } \\
\text { focus) }\end{array}$
\end{tabular}

$\begin{array}{ll}0.3 \text { Injection of ICG } & \text { Intradermal injection in first (ulnar injection Time of injection } \\ & \text { point) and fourth web space (radial injection } \\ & \text { point) dorsally in the hand } \\ & 0.2 \mathrm{~mL} \text { of the diluted solution is injected in } \\ & \text { each injection point }\end{array}$

Early phase $\quad 1.1$ Rest: $1 \mathrm{~min} \quad$ Hand in resting position on table

Linear transport starting from ulnar injection point: Yes / No (if 'yes', after ...... sec) Linear transport starting from radial injection point: Yes / No (if 'yes', after ...... sec)

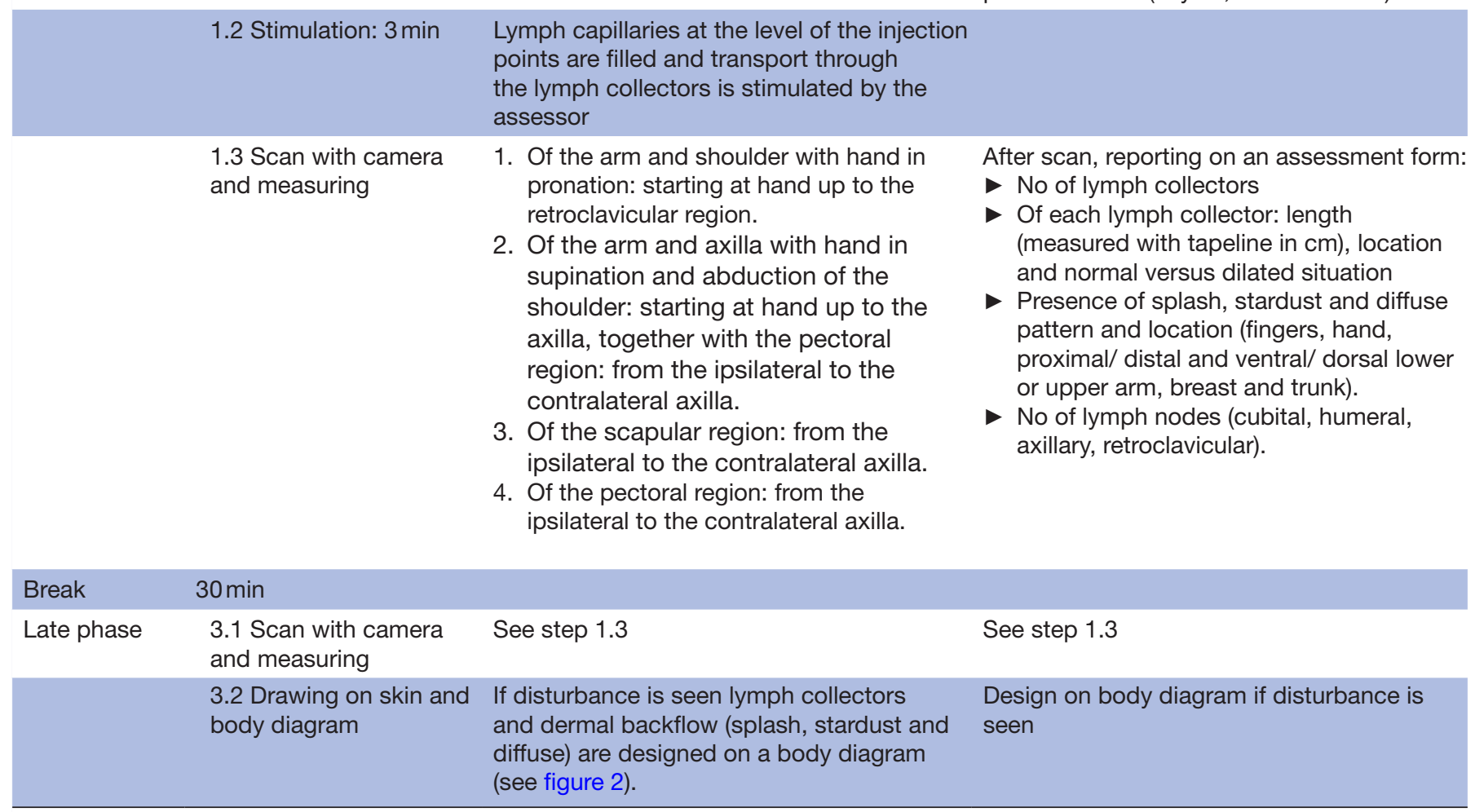

ICG, indocyanine green.

according to 'www.randomization.com'. This generator randomises each subject to a single treatment by using the method of randomly permuted blocks. Assessments are performed by a person blinded to the treatment allocation groups.

\section{Interventions}

During hospitalisation, all participants receive information about the prevention of lymphoedema. They are advised to avoid lifting heavy objects, but to use the affected arm as normally as possible. Limb constriction and extremes of temperatures should be avoided. In case of heaviness, the arm should be elevated. Skin care is recommended, and gain in body weight should be avoided to prevent lymphoedema. Patients receive a brochure which outlines these guidelines.

Participants are prescribed exercise therapy, which is started during hospitalisation with low level mobilising exercises for the hand, elbow and shoulder. After hospitalisation, these exercises are continued. Patients who underwent ALND are going to a physical therapist nearby to continue physical therapy such as passive mobilisation of the shoulder, stretching and transverse strain of the breast muscles, scar tissue massage and active mobilising and stabilising exercises. This starts twice a week and frequency is gradually diminished. Exercises are continued until a full range of motion is reached. When a seroma is present intensity of exercises is diminished. 

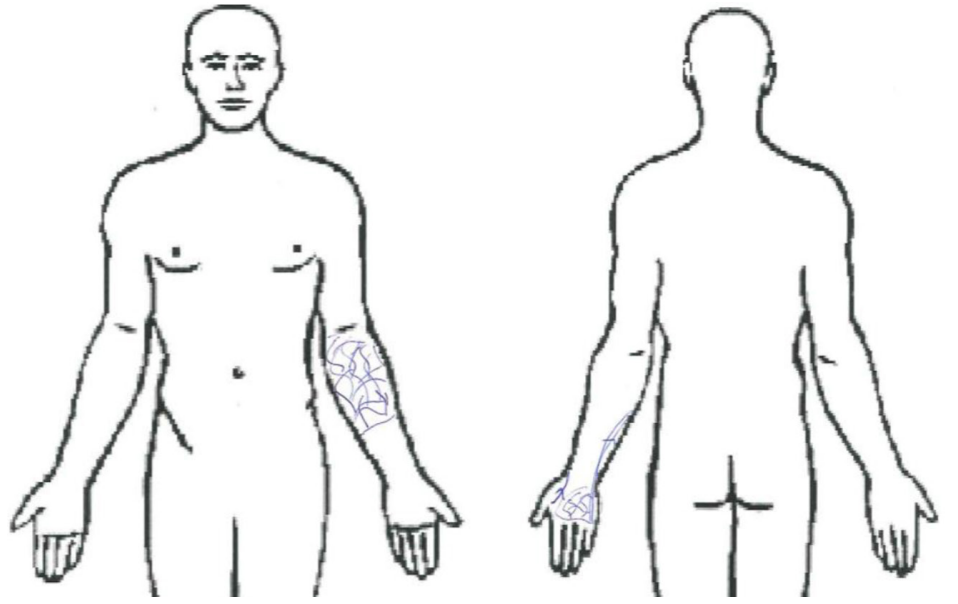

Legend:

-: place of injection

o: lymph node

$\longrightarrow$ : linear (lymph collector)

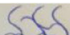

splash (lymph capillary)

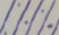

: stardust (lymph precollector)

XXY): diffuse (interstitial space)

Figure 2 Example of body diagram.

Patients who underwent SNB are not routinely seen by a physical therapist after discharge. If functional shoulder problems are seen at discharge or at follow-up consultation, physical therapy is prescribed. Patients are encouraged to do exercises at home twice a day until full range of motion is reached.

If early disturbance is seen on lymphofluoroscopy at a control visit, the patient is randomised in either the preventive treatment group or the control group. In the control group, the usual care is continued consisting of preventive measures and exercises as described above. The participants in the preventive treatment group receive the usual care and a compression garment whether or not combined with a glove on top. The compression garment is measured by an experienced compression specialist. The first choice is a round knitted custom-made compression garment, compression class $2(23-32 \mathrm{~mm} \mathrm{Hg})$. If patients are not comfortable with this garment, a flat-knitted garment is ordered. If the hand shows swelling after wearing the garment, a glove is measured. Patients need to wear the garment/glove at daytime during the remaining follow-up time of the trial. Written instructions for washing and maintenance of the garment and glove are given. Patients receive a new garment/glove every 6 months. A compression questionnaire is filled in at every visit to assess adherence and adverse events (AEs) of the compression material.

Table 2 Overview of measurement method and calculation of the primary outcomes

Outcome parameter Measurement time, method, material Calculation

Cumulative incidence of clinical Before surgery, at 12 months, 18 months, 24

lymphoedema defined as: months and 36 months.

$\geq 5 \%$ increase of relative arm volume difference compared with presurgical value
With perimeter

Circumferences at olecranon and 4, 8, 12, 16 and $20 \mathrm{~cm}$ above and under olecranon of arm at affected and healthy side ${ }^{12}$

With volumeter, weighing balance and recipient Water displacement method hand ${ }^{1314}$
$0=$ No clinical lymphoedema

$1=$ Clinical lymphoedema

Relative arm volume difference compared with presurgical value=relative arm volume difference at assessment - relative arm volume difference at baseline

Relative arm volume difference $=($ absolute arm volume difference/ arm volume healthy side) $\times 100$

Absolute arm volume difference $=$ arm vol affected side - arm volume healthy side

Arm volume =sum of volume of different arm segments determined by circumference measurements+hand vol Arm segment $=4 \times\left(\mathrm{C}_{1}^{2}+\mathrm{C}_{1} \mathrm{C}_{2}+\mathrm{C}_{2}{ }^{2}\right) / 12 \pi$, where $\mathrm{C}_{1}$ is the upper circumference and $\mathrm{C}_{2}$ is the lower circumference of each segment (formula of the truncated cone) ${ }^{12}$

Hand volume=volume measured with volumeter

\begin{tabular}{|c|c|c|}
\hline \multirow{2}{*}{$\begin{array}{l}\text { Proportion of subjects with } \\
\text { deterioration of the dermal } \\
\text { backflow }\end{array}$} & \multicolumn{2}{|l|}{$\begin{array}{l}\text { At } 12 \text { months, } 18 \text { months, } 24 \text { months and } 36 \\
\text { months. }\end{array}$} \\
\hline & $\begin{array}{l}\text { With lymphofluoroscopy: injecting ICG in the hand } \\
\text { of the affected arm, }{ }^{26} \text { protocol see table } 1\end{array}$ & $\begin{array}{l}0=\text { Stabilisation or improvement } \\
1=\text { Deterioration } \\
\text { Stabilisation: stable area of dermal backflow OR stable dermal } \\
\text { backflow pattern } \\
\text { Improvement: diminished area of dermal backflow OR } \\
\text { diminished severity of dermal backflow pattern } \\
\text { Deterioration: increased area of dermal backflow OR increased } \\
\text { severity of dermal backflow pattern }\end{array}$ \\
\hline
\end{tabular}

ICG, indocyanine green 


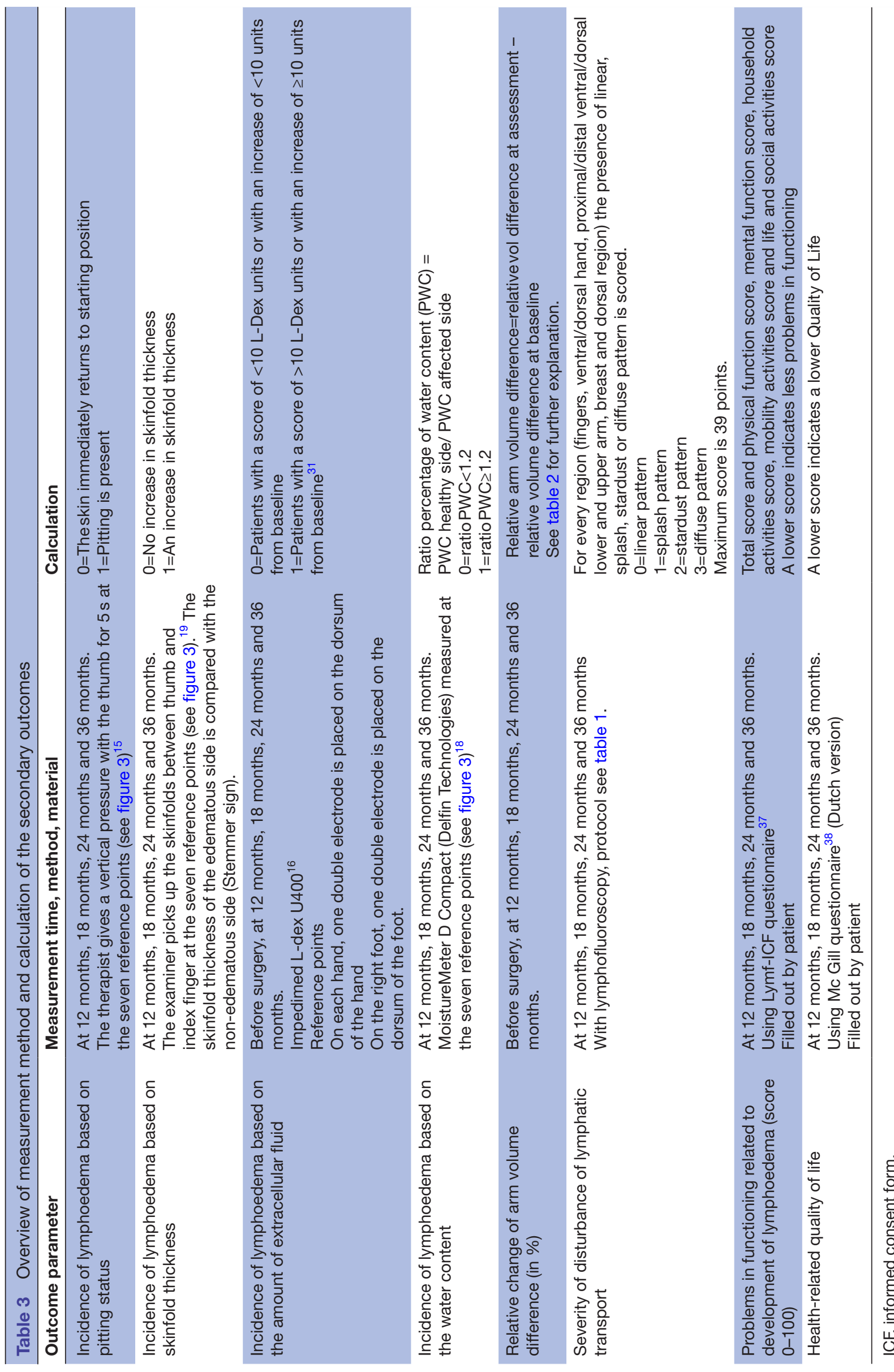




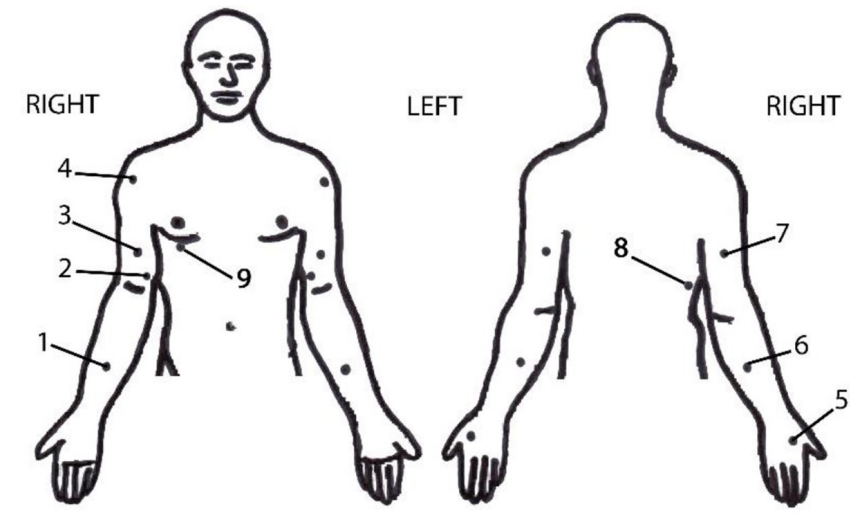

1. Ventral side forearm:

$15 \mathrm{~cm}$ distally of the elbow crease

2. Medial side upper arm:

$3 \mathrm{~cm}$ proximally of the medial epicondyle of the humerus

3. Ventral side upper arm:

$7 \mathrm{~cm}$ proximally of the elbow crease

4. Shoulder:

$5 \mathrm{~cm}$ distally of the lateral border of the acromion

in the direction of the lateral epicondyle of the humerus

5. Hand:

Central point between dorsal side thumb and index finger

6. Dorsal side forearm:

$10 \mathrm{~cm}$ distally of the proximal border of the olecranon

7. Dorsal side upper arm:

$7 \mathrm{~cm}$ proximally of the proximal border of the olecranon

8. Trunk:

$5 \mathrm{~cm}$ distally of the dorsal axillary fold

9. Breast:

Lumpectomy: $3 \mathrm{~cm}$ distally of the nipple (in case of

mastectomy: $3 \mathrm{~cm}$ distally from the middle of the scar)

Figure 3 Description of the reference points needed for the local clinical assessments.

If clinical lymphoedema is established the patient receives the normal standard of care treatment for lymphoedema with decongestive lymphatic therapy. Patients are referred to a specialised physical therapist or to the UZ Leuven center for lymphoedema.

\section{Primary outcomes}

The primary outcomes are the incidence of clinical lymphoedema of the arm/hand measured by circumference measurements and volume displacement defined as $5 \%$ vol increase compared with the contralateral side (first primary outcome) and the proportion of subjects with deterioration of the DB measured by lymphofluoroscopy (second primary outcome) (see table 2 ).

\section{Secondary outcomes}

Secondary outcome measures are: the incidence of clinical lymphoedema of the arm/hand based on the extracellular fluid content, based on the water content, based on thickening of the skinfold, the relative change of arm volume, the severity of disturbance of lymphatic transport, the change in functional problems related to the lymphoedema and the change in health-related quality of life (see table 3).

\section{Sample size calculation}

For both hypotheses a sample size calculation is performed.

For the hypothesis that the incidence rate of clinical lymphoedema will be lower in the preventive treatment group than in the control group, we estimate that $50 \%$ of the patients in the control group will develop clinical lymphoedema in the first year after the randomisation compared with $5 \%$ in the preventive treatment group (wearing a compression garment). The $5 \%$ is based on previous studies. ${ }^{30-32}$ A study of Stout $e t a l^{30}$ treated patients, diagnosed with subclinical lymphoedema, defined as a volume difference between both limbs of $\geq 3 \%$, with a compression garment. The incidence of lymphoedema (stage I/II) at 5 years was $5.6 \%$. Another trial showed that the same type of treatment reduced the incidence of lymphoedema to $4.4 \% .{ }^{31}$ The $50 \%$ incidence of clinical lymphoedema in the control group is based on expert opinion.

The sample size calculation is based on the formula in Diggle for a longitudinal study for showing a timeaveraged treatment effect for a binary outcome. Four time points per patient are foreseen (12 months, 18 months, 24 months, 36 months). Conservatively a high correlation of 0.90 between repeated measurements is assumed (higher correlation means larger sample size). Based on a power of $80 \%$ and $2.5 \%$ significance level (with a Bonferroni correction for multiple testing given that we test two outcomes, and keeping a family-wise alpha of $5 \%$ ), we need a sample size of 14 patients per group. Taking into account a drop-out rate of $10 \%, 16$ patient per group or a total of 32 randomised patients are needed.

For the hypothesis that patients in the preventive treatment group will have less deterioration of DB visualised by lymphofluoroscopy, we estimate that a deterioration of the DB can be expected in $40 \%$ of the cases in the preventive treatment group in contrast to $90 \%$ in the control group. There is one publication studying early detection with lymphofluoroscopy and the changes of the DB pattern in case of early treatment. Therapy consists of exercise, skin care, elevation and the use of a compression garment. This trial shows that only three out of 35 patients with DB deteriorate during the follow-up. ${ }^{29}$ Deterioration was described as a change in severity of the DB pattern. In our study also the area of $\mathrm{DB}$ is taken into account, therefore, we estimate a higher rate of deterioration. The $90 \%$ deterioration in the control group is based on expert opinion. The analysis is performed on a binary response (worsening vs stable condition/ improvement). Sample size calculation is completely analogous to the first outcome, leading to a total sample size of 30 patients after taking into account $10 \%$ of drop-out.

To calculate the total amount of patients to be included in the present trial two prospective observational studies about the incidence of subclinical lymphoedema where considered $^{30-32}$ and one study about lymphofluoroscopic observations. $^{29}$ In the study by Akita et al, 196 patients are included in a 1-year follow-up study with 
lymphofluoroscopy. Twenty-five per cent of the patients developed a DB pattern on lymphofluoroscopy. ${ }^{29}$ The largest of both sample sizes, that is, 32 patients, is adopted. We estimate that in $25 \%$ of the patients an early disturbance will be seen, hence 128 patients are included in the trial.

\section{Statistical methods}

Logistic regression analysis will be used for both primary endpoints, studying the difference between the preventive treatment and control group over the follow-up period. Generalised estimating equations are used to account for repeated measurement. Model covariates include time and treatment group. The main effect of the preventive treatment group is estimated and presented by ORs with $95 \%$ CIs. Both analyses are tested at the $2.5 \%$ significance level.

All data are analysed according the intention to treat principle.

A 5\% level of significance is applied for all secondary analyses.

\section{Monitoring}

There are no indications for setting up a data monitoring committee.

No AEs are expected. AE will be reported during the entire trial period, that is, 36 months.

It will be specified that the investigator(s) and the institution(s) will permit trial-related monitoring, audits, Ethical Committee review and regulatory inspections (where appropriate) by providing direct access to source data and other documents (ie, patients' case files).

\section{DISCUSSION}

This is the first randomised controlled clinical trial investigating the additional effect of wearing a compression garment, to the usual care (ie, information and exercises), on the incidence of clinical lymphoedema and/ or deterioration of the DB visualised by near infrared fluorescence imaging, in patients with early disturbance of the lymphatic transport (ie, DB) after treatment for breast cancer. If treatment can start in this early phase of disturbance, further evolution to clinical lymphoedema can perhaps be prevented.

\section{ETHICS AND DISSEMINATION}

The trial is conducted in compliance with the principles of the Declaration of Helsinki (2008), the principles of Good Clinical Practice and in accordance with all applicable regulatory requirements. This protocol and related documents has been approved by the Ethical Committee of the University Hospitals Leuven (CME reference S60382, EudraCT Number 2017-002306-12).

The study can and will be conducted only on the basis of prior informed consent by the participants, or their legal representatives, to participate in the study. The investigator will obtain a signed informed consent form (ICF) for all patients prior to their enrolment and participation in the study in compliance with all applicable laws, regulations and the approval of the ethics committee. The investigator will retain such ICFs in accordance with the requirements of all applicable regulatory agencies and laws.

The investigator will treat all information and data relating to the study disclosed as confidential and shall not disclose such information to any third parties or use such information for any purpose other than the performance of the study. The collection, processing and disclosure of personal data, such as patient health and medical information is subject to compliance with applicable personal data protection and the processing of personal data (Directive 95/46/EC and Belgian law of 8 December 1992 on the Protection of the Privacy in relation to the Processing of Personal Data).

Data are anonymous if no one, not even the researcher, can connect the data to the individual who provided it. No identifying information is collected from the individual.

When data are coded, there continues to be a link between the data and the individual who provided it. The research team is obligated to protect the data from disclosure outside the research according to the terms of the research protocol and the informed consent document. The subject's name or other identifiers is stored separately (site file) from the research data and replaced with a unique code to create a new identity for the subject. The data are stored on a shared file. Only the principal investigator, subinvestigators and project coworkers (after permission from the principal investigator) have access to the patient file.

The authors declare that they have no known competing financial interests or personal relationships that could have appeared to influence the work reported in this paper.

The results of the study will be send for publication to a peer-review journal. Participants and healthcare providers will be invited for a symposium to communicate the trial results.

\section{Author affiliations}

${ }^{1}$ Department of Vascular Surgery, University Hospitals Leuven, Leuven, Belgium ${ }^{2}$ Department Cardiovascular sciences, Research unit Vascular Surgery, KU Leuven University of Leuven, Leuven, Belgium

${ }^{3}$ Department of Rehabilitation Sciences, KU Leuven - University Hospitals of Leuven, Leuven, Belgium

${ }^{4}$ Multidisciplinary Breast Centre, University Hospitals Leuven, Leuven, Belgium

Acknowledgements The authors are very grateful to the Multidisciplinary Breast Clinic for collaborating in this study. The authors also extend very grateful thanks to the study participants and the patients who were involved in the design of the study.

Contributors ST drafted the manuscript. ST is the principal investigator of the DEARLY trial. BB-H, IF, ND, IN and PN contributed substantially to the establishment of the protocol, revised the manuscript for important intellectual content and provided input according to their area of expertise. All authors approved the final version and agreed to be accountable for all aspects of the work in ensuring that questions related to the accuracy or integrity of any part of the work are appropriately investigated and resolved. 
Funding The Dearly trial is financed by the Clinical Research Fund of the University Hospitals Leuven, K00R 2017, S60382.

Competing interests None declared.

Patient and public involvement Patients and/or the public were involved in the design, or conduct, or reporting, or dissemination plans of this research. Refer to the Methods section for further details.

Patient consent for publication Not required.

Provenance and peer review Not commissioned; externally peer reviewed.

Open access This is an open access article distributed in accordance with the Creative Commons Attribution Non Commercial (CC BY-NC 4.0) license, which permits others to distribute, remix, adapt, build upon this work non-commercially, and license their derivative works on different terms, provided the original work is properly cited, appropriate credit is given, any changes made indicated, and the use is non-commercial. See: http://creativecommons.org/licenses/by-nc/4.0/.

ORCID iD

Sarah Thomis http://orcid.org/0000-0002-8491-6264

\section{REFERENCES}

1 Executive Committee of the International Society of Lymphology. The diagnosis and treatment of peripheral lymphedema: 2020 consensus document of the International Society of Lymphology. Lymphology 2020;53:3-19.

2 Morgan PA, Franks PJ, Moffatt CJ. Health-Related quality of life with lymphoedema: a review of the literature. Int Wound J 2005;2:47-62.

3 Saito Y, Nakagami H, Kaneda Y, et al. Lymphedema and therapeutic lymphangiogenesis. Biomed Res Int 2013;2013:1-6.

4 DiSipio T, Rye S, Newman B, et al. Incidence of unilateral arm lymphoedema after breast cancer: a systematic review and metaanalysis. Lancet Oncol 2013;14:500-15.

5 Gebruers N, Verbelen H, De Vrieze T, et al. Incidence and time path of lymphedema in sentinel node negative breast cancer patients: a systematic review. Arch Phys Med Rehabil 2015;96:1131-9.

6 Rockson SG. Precipitating factors in lymphedema: myths and realities. Cancer 1998;83:2814-6.

7 Sun F, Skolny MN, Swaroop MN, et al. The need for preoperative baseline arm measurement to accurately quantify breast cancerrelated lymphedema. Breast Cancer Res Treat 2016;157:229-40.

8 Carlson JA. Lymphedema and subclinical lymphostasis (microlymphedema) facilitate cutaneous infection, inflammatory dermatoses, and neoplasia: a locus minoris resistentiae. Clin Dermatol 2014;32:599-615.

9 Michelini S, Campisi C, Gasbarro V, et al. National guidelines on lymphedema. Lymphology 2007;55:238-42.

10 International Society of Lymphology. The diagnosis and treatment of peripheral lymphedema: 2013 consensus document of the International Society of Lymphology. Lymphology 2013;46:1-11.

11 De Vrieze T, Gebruers N, Tjalma WA, et al. What is the best method to determine excessive arm volume in patients with breast cancerrelated lymphoedema in clinical practice? Reliability, time efficiency and clinical feasibility of five different methods. Clin Rehabil 2019;33:1221-32.

12 Devoogdt N, Lemkens H, Geraerts I, et al. A new device to measure upper limb circumferences: validity and reliability. Int Angiol 2010;29:401-7.

13 Gebruers N, Truijen S, Engelborghs S, et al. Volumetric evaluation of upper extremities in 250 healthy persons. Clin Physiol Funct Imaging 2007;27:17-22.

14 Damstra RJ, Glazenburg EJ, Hop WCJ. Validation of the inverse water volumetry method: a new gold standard for arm volume measurements. Breast Cancer Res Treat 2006;99:267-73.

15 Sanderson J, Tuttle N, Box R, et al. The pitting test; an investigation of an unstandardized assessment of lymphedema. Lymphology 2015;48:175-83.

16 Cornish B. Bioimpedance analysis: scientific background. Lymphat Res Biol 2006;4:47-50.
17 Mayrovitz HN, Weingrad DN, Davey S. Local tissue water in at-risk and contralateral forearms of women with and without breast cancer treatment-related lymphedema. Lymphat Res Biol 2009;7:153-8.

18 Mayrovitz HN, Weingrad DN, Lopez L. Tissue dielectric constant (TDC) as an index of skin water in women with and without breast cancer: upper limb assessment via a self-contained compact measurement device. Lymphology 2016;49:27-35.

19 Brorson H. Liposuction in arm lymphedema treatment. Scand J Surg 2003:92:287-95.

20 Kinmonth JB, Taylor GW, Tracy GD, et al. Primary lymphoedema; clinical and lymphangiographic studies of a series of 107 patients in which the lower limbs were affected. Br J Surg 1957;45:1-10.

21 Henze E, Schelbert HR, Collins JD, et al. Lymphoscintigraphy with Tc-99m-labeled dextran. J Nucl Med 1982;23:923-9.

22 Szuba A, Shin WS, Strauss HW, et al. The third circulation: radionuclide lymphoscintigraphy in the evaluation of lymphedema. $J$ Nucl Med 2003;44:43-57.

23 Rasmussen JC, Tan I-C, Marshall MV, et al. Human lymphatic architecture and dynamic transport imaged using near-infrared fluorescence. Transl Oncol 2010;3:362-7.

24 Tan I-C, Maus EA, Rasmussen JC, et al. Assessment of lymphatic contractile function after manual lymphatic drainage using near-infrared fluorescence imaging. Arch Phys Med Rehabil 2011;92:756-64.

25 Abbaci M, Conversano A, De Leeuw F, et al. Near-Infrared fluorescence imaging for the prevention and management of breast cancer-related lymphedema: a systematic review. Eur J Surg Oncol 2019;45:1778-86.

26 Yamamoto T, Yamamoto N, Doi K, et al. Indocyanine green-enhanced lymphography for upper extremity lymphedema: a novel severity staging system using dermal backflow patterns. Plast Reconstr Surg 2011;128:941-7.

27 Mihara M, Hara H, Araki J, et al. Indocyanine green (ICG) lymphography is superior to lymphoscintigraphy for diagnostic imaging of early lymphedema of the upper limbs. PLoS One 2012;7:e38182-9.

28 Akita S, Mitsukawa N, Kazama T, et al. Comparison of lymphoscintigraphy and indocyanine green lymphography for the diagnosis of extremity lymphoedema. Journal of Plastic, Reconstructive \& Aesthetic Surgery 2013;66:792-8.

29 Akita S, Nakamura R, Yamamoto N, et al. Early detection of lymphatic disorder and treatment for lymphedema following breast cancer. Plast Reconstr Surg 2016;138:192e-202.

30 Stout Gergich NL, Pfalzer LA, McGarvey C, et al. Preoperative assessment enables the early diagnosis and successful treatment of lymphedema. Cancer 2008;112:2809-19.

31 Soran A, Ozmen T, McGuire KP, et al. The importance of detection of subclinical lymphedema for the prevention of breast cancerrelated clinical lymphedema after axillary lymph node dissection; a prospective observational study. Lymphat Res Biol 2014;12:289-94.

32 Shah C, Arthur DW, Wazer D, et al. The impact of early detection and intervention of breast cancer-related lymphedema: a systematic review. Cancer Med 2016;5:1154-62.

33 Gençay Can A, Ekșioğlu E, Çakçı FA. Early detection and treatment of subclinical lymphedema in patients with breast cancer. Lymphat Res Biol 2019;17:368-73.

34 Hayes SC, Johansson K, Stout NL, et al. Upper-body morbidity after breast cancer: incidence and evidence for evaluation, prevention, and management within a prospective surveillance model of care. Cancer 2012;118:2237-49.

35 Armer JM, Stewart BR. A comparison of four diagnostic criteria for lymphedema in a post-breast cancer population. Lymphat Res Biol 2005;3:208-17.

36 Czerniec SA, Ward LC, Kilbreath SL. Breast cancer-related arm lymphedema: fluctuation over six months and the effect of the weather. Lymphat Res Biol 2016;14:148-55.

37 Devoogdt N, Van Kampen M, Geraerts I, et al. Lymphoedema functioning, disability and health questionnaire (Lymph-ICF): reliability and validity. Phys Ther 2011;91:944-57.

38 De Vrieze T, Coeck D, Verbelen H, et al. Cross-Cultural psychometric evaluation of the Dutch McGill-QoL questionnaire for breast cancer patients. Facts Views Vis Obgyn 2016;8:205-9. 